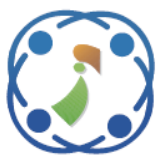

\title{
Urgency Zone Partitioning-Based Data Diffusion Model for Intelligent Transport Systems
}

\author{
Kalaivani Dhanasegaran ${ }^{1 *}$ \\ Rajkumar Soundrapandiyan ${ }^{2}$ \\ ${ }^{1}$ Department of Information Technology, \\ CMR Institute of Management Studies (Autonomous), Bangalore, Karnataka, India \\ ${ }^{2}$ School of Computer Science and Engineering, \\ Vellore Institute of Technology, Vellore, Tamil Nadu, India \\ *Corresponding Author Email: kalaivanidhanasegaran0904@gmail.com
}

\begin{abstract}
These days, road safety applications have become significant due to the assurance of Quality of Service (QoS) requirements such as scalability, latency, and security facilitates multi-hop wireless broadcast capabilities in VANETs. Factors such as limited bandwidth, minimum contention window size and mobility of vehicular nodes make multi-hop broadcast a challenging endeavor. This paper proposes a suitable data diffusion model with emergency message transfer and clustering of neighborhood nodes based on zones. Based on the location of emergency-message senders, zones such as Red Orange and Yellow (ROY) are created to partition the vehicle in terms of directional, bi-directional and multi-directional broadcast in diverse directions. The adjustments in urgency factor on the basis of the emergency seamlessly handle the traffic target areas. The analysis and simulation result shows that the proposed diffusion model improves the performance aspects such as delay, message overhead and reception rate compared to the existing models.
\end{abstract}

Keywords: Directional broadcast, Emergency message, Mobility, Quality of service (QoS), Vehicular ad-hoc network (VANET).

\section{Introduction}

In recent times, the focus on global transport system has shifted towards vehicle to vehicle communication since light vehicles are equipped with wireless communication. These networks enable risk reduction and improvement of security performance. The realization of Vehicular Ad-hoc Networks (VANETs) comprises vehicle communication protocols. The major objective of VANET technology is to broadcast the safety message among all nodes via either vehicle or road safety unit. The prior information broadcast from halting vehicle to nearby vehicles avoid collision and warns the driver to optimize the speed. Based on the instant of broadcast, the messages are classified into two categories namely periodic and event driven. In case of the former, the messages get broadcasted automatically amongst the nodes under periodical manner. Whereas in latter, the messages are broadcasted in hazardous conditions. Minor failures in the aforementioned broadcasting messages might result in drastic consequences. The utilization of wireless access technology ensures reliable broadcast service. However, broadcast is such a crucial task in high-priority safety networks and achieving QoS requirements assumes paramount significance. Hence, the shift of focus of research towards the advancement of wireless standards such as IEEE $802.11 \mathrm{p}$ with the Time Division Multiple Access (TDMA) protocol (VeMAC) [1]. The major limitation of such a network is the lack of proper acknowledgement message in packet delivery and the probability of failure in transmissions in small contention window.

The safety contents by Road Side Units (RSU) utilize On-Board Units (OBUs) with geographical maps commonly referred as popular content 
distribution. Peer-to-Peer (P2P) protocols have been employed to download various multimedia services to support efficient Persistence Contrastive Divergence (PCD).The adaptation of P2P ideas to construct an efficient Vehicle-to-Vehicle (V2V) communication in VANET usually consumes less than one minute. However, download of a large file by the OBUs within the duration of one minute is impossible and hence monitoring of packet exchange amongst the OBUs is necessary for the following scenarios [2]:

- Unreliable wireless links due to the cochannel interference

- Unpredictable network topology due to highmobility nodes

The incorporation of Cognitive Radio (CR) with the coalitional graph and game theory addresses the above issues to improve the self-organizing capabilities of VANETs. The mitigation in selective frequency multipath fading, Inter-Symbol Interference (ISI) to achieve high-spectral efficiency in IEEE $802.11 \mathrm{p}$-based V2V communication requires the Orthogonal Frequency Division Multiplexing (OFDM) and its performance depends on the subcarriers. The elimination of subcarriers requires the Carrier Frequency Offset (CFO) which leads to the Inter-Carrier Interference (ICI) [3]. High-doppler shifts, rapid movements of transmitter (TX) and receiver (RX) make the ICI problem as more difficult that leads to ICI cancellation scheme. The utilization of the two isolated OFDM transceivers requires the implementation of two-path cancellation scheme which leads to high realization complexities.

The participation of numerous of vehicle nodes increases the count of transmit/receive packets which consume more bandwidth and delay in transmission. Besides, the mobility of nodes induces the packet loss and security issues. The improper disclosure of vehicle location information and the modification of information by the malicious user are the major deficiencies during the exchanges of packets. To alleviate such issues, the contact level or the social level schemes are employed to enhance the communication efficiency and reduce the transmission delay effectively. Hence, research turns into the data dissemination approach or gathering approach through the clustering of vehicle nodes to assure the privacy protection scheme. The cooperative downloading strategy [4] with the use of Vehicle to Information unit (V2I) and V2V communication improves the throughput level without considering the delivery delay. Hence, the next generation transport systems focus on the reduction of delivery delay and improvement in downloading throughput.

The characterization of VANET by the timevarying topology utilizes the random-channel access protocol which suffers from number of issues namely, collision random interaction or decoding status. To alleviate such issues, the data dissemination schemes adopt the scheduling strategy namely, Space-Time Network Coding (STNC) [5]. In this scheme, the scheduling of transmission and avoidance of collision are performed and controlled by the Central Server (CS). The modification of random access scheme by the scheduling strategy makes the RSU as reasonable units for topology monitoring. Here, the CS selects the nodes for participating in the communication and assigns the transmission frames. Practically, the data dissemination utility suffers from the constant changes during the switch over from one transmission frame to next. Hence, the proper relay selection algorithm is used to select the suitable node within the maximum data dissemination utility for each frame. This paper proposes the data diffusion model to address the aforementioned issues with the three schemes: labeled messages generation on the basis of urgency, capability of each vehicle to change the urgency factor within the zone of near vehicles and the creation of partition zones namely ROY model. The novel technical contributions of proposed diffusion model are listed as follows:

- The generation of labelled messages on the basis of urgency factor is helpful to efficiently disseminate the emergency message to the nearby vehicles in VANETs

- The partitioning of road into three zones such as ROY from the accident zone supports the efficient emergency message delivery through the pre-planned time slots

- The clustering of vehicles in the single hop range and the broadcast of emergency messages based on the priority level reduces the unnecessary delay and improves the accurate delivery of emergency message to the target area.

The paper organized as follows: The detailed description of the related works on data dissemination models to analyze the traffic scenarios under emergency condition are discussed in section II. The implementation process of Urgency Zone Partitioning-based Data Diffusion (UZPDD) model for emergency message delivery analysis is described in section III. The comparative analysis of proposed UZPDD with existing methods provided in 
section IV. Finally, the conclusion about the application of UZPDD on the large size data is presented in section $\mathrm{V}$.

\section{Related work}

In this section, the existing techniques and protocols used for enabling a reliable data transmission in VANET are investigated with its benefits and demerits. . Luan et al. [1] focused the improvement of Distributed Coordinated Function (DCF) performance with the consideration of mobility of the nodes. Typically, the rendering of stations in an opportunistic manner through the utilization of DCF with the cherished access time of the users was highly useful to increase the system throughput. Here, the Markovian chain model was utilized to integrate the vehicles and to reduce the negative effects with improved system throughput. But in wireless medium, transmission is not efficient. The segment creation based on the urgency and the data forwarding among the nearest vehicles were the major issues in the VANETs. Sahoo et al. [2] implemented an IEEE 802.11 multi-hop broadcast protocol for improving the performance of VANET, where the binary partition based approach was utilized to split the network area inside the transmission range into the number of segments with reduced delay. Even it is a single type of traffic in the network and cannot address the issues clearly. The individual data transmission among the segments with the above mechanism utilizes the different V2I and V2V wireless links to access the same resources which generated the resource sharing problem. Zhang et al. [3] investigated this resource sharing problem by applying the graphtheory model and the interference resource sharing schemes in two aspects: interference aware graphbased resource sharing and the interference classified graph-based resource sharing schemes. The overall network sum rate was improved with the resource sharing schemes compared to existing mechanisms effectively and thus consists of a critical challenge.

The evolution of parameter computation methods addressed the issues in achieving the accuracy-efficiency trade off to facilitate the design and performance analysis of $\mathrm{V} 2 \mathrm{~V}$ communication. Cheng et al. [4] introduced an improved version of Modified Method of Equal Area Access (IMMEA) for designing the deterministic channels. Also, the authors provided some sufficient approximations for analyzing the statistical properties without affecting the simulation efficiency. The authors [5] considered the major statistical properties of Level
Crossing Rate (LCR) and Average Fade Duration (AFD) by using the new model called geometrybased stochastic model for non-isotrophic scattering $\mathrm{V} 2 \mathrm{~V}$ communication. In addition to these, the shape of the region, mean angle directions in which TX and RX are moved were considered to investigate the impact of $\mathrm{V} 2 \mathrm{~V}$ density through the Ricean Fading channels.

The advantages of the collaboration schemes were inherited into $\mathrm{V} 2 \mathrm{~V}$ communication to improve the safety and comfort level of the participants. Tornell et al. [6] presented the driving safety application on the basis of the dissemination of warnings of the smart phone models through the enhanced Message Dissemination based on Roadmaps (eMDR) protocol. The integration of navigation system with the smart phones is helpful to track their location and route information alternate route suggestion during the emergency condition. The feasibility of the eMDR protocol was analyzed under diverse conditions with the integration of wireless channel maps and GPS location services. During the dissemination, the reduction of dissemination time and the improvement in number of vehicles to get the safety alert messages were the necessary objectives in VANETs. Fogue at al. [7] investigated the data dissemination strategies in the real urban scenarios through the scheme called Profile-driven Adaptive Warning Dissemination Scheme (PAWDS). This scheme considered the characteristics of the street area and the density of vehicles moving across the area through the mapping technique to improve the alert dissemination performance. But it is not incorporated with multicast protocols and number of messages received per vehicle is notably rises. Even though the dissemination schemes offered the better performance in road safety applications, the satisfaction level of the QoS requirements was minimum level. Latency, reliability and security are the major QoS requirements in urban transportation development. Bi et al. [8] proposed an Urban Multihop Broadcast Protocol (UMBP) to disseminate the emergency message $\mathrm{s}$ with minimum delay and redundancy. They compared the performance of UMBP with the existing Binary-Partition-Assisted Broadcast (BPAB) [2]. The forwarding mechanism divided the network into various partition such as mini-slot and the black-burst to select the neighbour nodes quickly. The application of the directed broadcast enabled the accurate delivery of emergency message to the target area with highperformance. The Intelligent Transportation System (ITS) is the crucial component of the modern society 


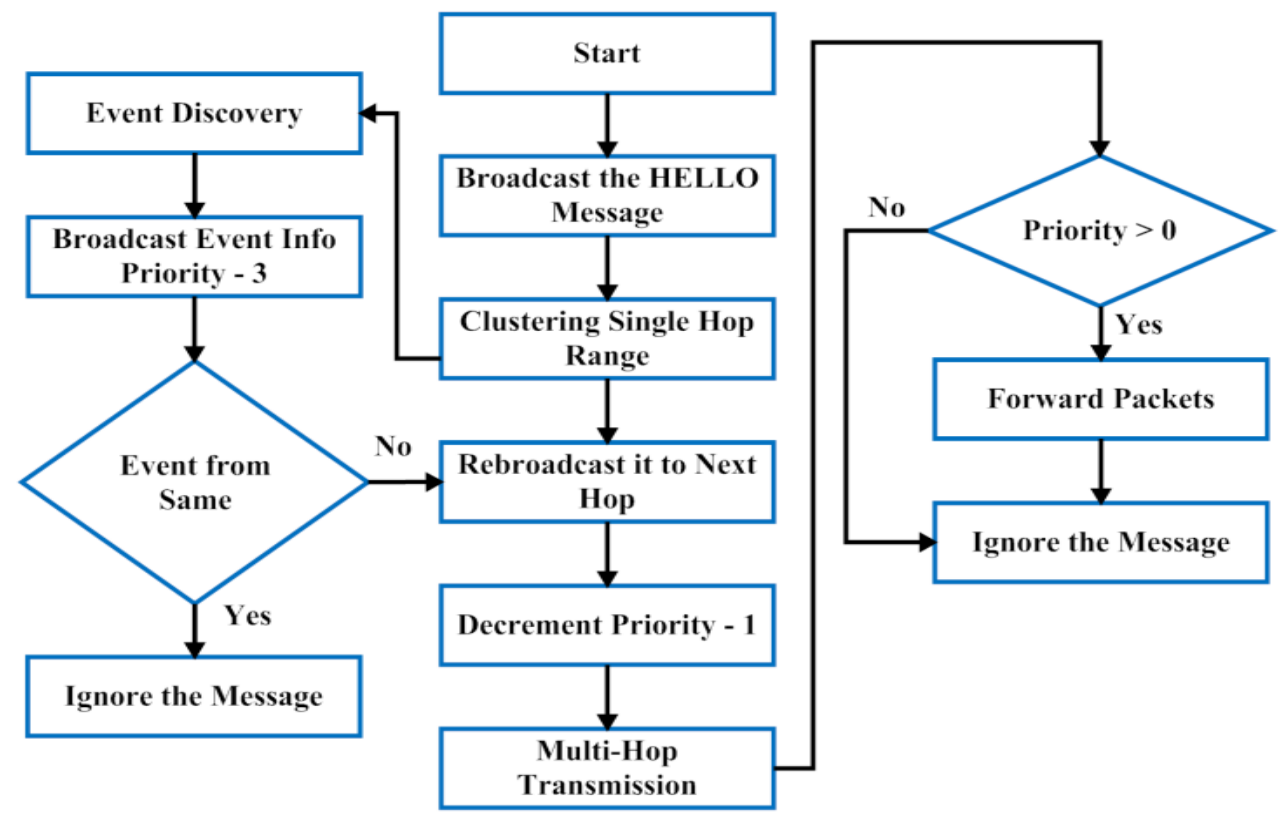

Figure. 1 Workflow of proposed UZPDD

which requires the interactions between the vehicles with the surrounding environment which leads to new opportunities according to the diverse applications with high-safety, security and efficiency management in the road safety applications. Cheng et al. [9] addressed the spectrum management problem with the device to device communication to address the challenges to assure the feasibility. They also proposed novel remedies which were necessary to make the Device-to-Device (D2D) as the practical for ITS.

Suthaputchakun et al. [10] proposed the multihop broadcast protocol to disseminate the critical emergency messages in IEEE 802.11p-based VANETs. They proposed the burst-based broadcast protocol with the two mechanisms as follows; introduction of interframe spaces in the Medium Access Control (MAC) layer to transfer the critical emergency message delivery with high-speed and the trinary partitioning for improvement of dissemination speed. The reduction in contention period resulted in more stable contention period that confirmed the effectiveness of broadcasting mechanisms effectively.

The safety related communication required the fast urgency-message delivery which demands the broadcast communication. Ma et al.[11] proposed the distributed cross layer scheme to design the control channel with the following processes: preemptive priority assignment and the dynamic receiver oriented packet repetitions along the interframe space. The robustness, scalability and the implementation processes were tested and the hidden terminal problem was analyzed in detail.
Omar et al. [12] introduced the VeMAC protocol on the basis of the TDMA and MAC mechanism to support one-hop and multi-hop broadcast services on the control channel through the acknowledgement schemes reduced the hidden terminal problem effectively. The assignment of disjoint sets of timeslots for the vehicles moving in opposite directions reduced the transmission collisions effectively. The comparison among the VeMAC with the ad-hoc MAC and TDMA confirmed the throughput performance. The authors [13] defined the working of VeMAC protocol on the periodic and event-driven safety messages to analyze the total delay for the delivery messages and the satisfaction of QoS by using the multiple time slots corresponding to different data units. The flexible utilization of time slots on the Control Channel $(\mathrm{CCH})$ was useful to support large size applications with minimum delay. The impact of traditional ad-hoc networks on the large scale VANETs was ineffective due to the rapid movement of vehicles. Cheng et al. [14] provided the comprehensive overview of radio channel access protocols and the resource management approaches with the deep investigation of suitability of the infotainment and safety service support on VANETs.

The provision of information regarding the road conditions under the weather scenario required the multimedia information. The development of interest ontology of cellular automata clustering with the new zone called zone of interest. The current study evaluated by using the extensive simulations and confirmed the effectiveness of CA clustering. 


\section{Urgency zone partitioning-based data diffusion model}

This section discusses the implementation details of the proposed Urgency Zone Partitioningbased Data Dissemination (UZPDD) for intelligent transport systems. The workflow of the proposed UZPDD is shown in Fig. 1.

The proposed workflow contains bass model to describe the interaction among the users and potential users. The incorporation of macro-level data diffusion model instead of micro-level model with the coefficient of innovation as the percentage of population. The dissemination of new product among the people is similar to the dissemination of emergency messages to the nearby vehicles to handle the traffic scenarios. Initially, the HELLO message is broadcasted among the devices in VANET. Then, the devices lies within the single hop range are clustered. During the clustering process, the event is discovered and broadcast such event information to validate the priority level. If the event arises from the same region, then ignore the message otherwise broadcast the information to the next hop. After that, the multi-hop transmission is initiated by decrementing the priority as 1 .If the priority level is greater than zero, then the packets are forwarded otherwise ignored the message. The validation of priority level and the message forwarding to the vehicles handles the traffic scenarios with minimum time effectively.

\subsection{System model}

The proposed system in this paper considers the general data dissemination model for VANET. The data source in this architecture is RSU and the vehicles are regarded as the end receivers or nodes. The RSU is connected to the core internet through the wired backhaul. Each vehicle in this architecture has the capability to share the data among the other vehicles and RSU. The population includes the set of vehicles with the wireless transceivers to communicate with the roadside infrastructures. In this environment, the vehicles that are involved in the wireless communication are considered as the input during initial population. Then, the initial region $\mathrm{t}$ is updated as 0 , and its time period may vary (i.e. $P(t)$ ) for each population. There are three parameters are considered for constructing the mobility model such as velocity, acceleration and deceleration speed. In this paper, the mobility model is considered as the macro model and utilizes the average speed for all the vehicles. Transmission range directly affects the alert message dissemination process. The basic assumption to propose the diffusion model is all the vehicles have the ability to communicate among them either directly or indirectly. This paper proposes the data diffusion model on the basis of urgency message transmission with the dissemination rate of message.

\subsection{Urgency message with time}

The occurrence of traffic accidents on the road or intersection point of urban areas initiates an emergency message. Based on such message, the road is partitioned into three namely Red Orange Yellow (ROY). The numerical value of alert message is high for $R$ zone since it is highly close to the accident. The value of alert message is medium in $O$-zone and the messages are not urgent in $Y$ zone. The number of vehicles has their own ROY model in the form of $(R, O, Y)$ which is not static due to the mobility of vehicles and the dynamics of traffic.

The length of the zone is dynamical which depends on the type of message, traffic density and speed of vehicle. In this paper, the zones are modelled as shown in Fig. 2.

In the ROY zone, the vehicles such as $B, C$, and $D$ are in the zone, then the vehicles $E, F, G$, and $H$ are present in the orange zone, and the vehicles $I$ and $J$ are present in the yellow zone. Here, the vehicle $B$ forwards the emergency message to the vehicles $C$ and $D$, then $D$ forwarded the message to its nearest node $E$ that forwards the message to the $F$, $G$, and $H$ based on this order the communication is enabled in this environment. Moreover, the forward data transmission only considered in this communication, so in the orange zone, the vehicle $D$ is not considered, because it creates the repetition during data transmission.

Let us considered the vehicle $A$ and the corresponding ROY model is expressed as $A_{R O Y}$ follows:

$$
\begin{aligned}
& A_{R}=(B, C, D) \\
& A_{O}=(E, F, G, H) \\
& A_{Y}=(I, J)
\end{aligned}
$$

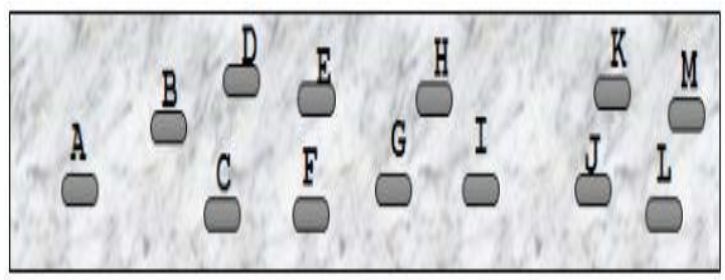

Figure. 2 Zone partitioning 
Similarly, the vehicle $E$ created its own model by $E_{R O Y}$

$$
\begin{aligned}
& E_{R}=(F, G, H) \\
& E_{O}=(I, J) \\
& E_{Y}=(K, L, M)
\end{aligned}
$$

Once the zones are separated, the diffusion model is constructed with the message value of messages and priority assignment.

\subsection{Diffusion model with value of messages}

The emergency or urgency message containing the zones such as ROY is disseminated among the nodes in various simulation periods. Under the first dissemination, the relationship between the density function with the parameters is used to construct the diffusion function.

$$
\begin{aligned}
& \frac{f(t)}{1-F(t)}=[p+q F(t)] x(t) \\
& x(t)=1+\beta \frac{V^{\prime}(t)}{V(t)}
\end{aligned}
$$

With the inclusion of percentage value of deduction per time units, the Eq. (8) modified as

$$
x_{1}(t)=1-\beta \frac{1}{\ln (1-\tau)}
$$

Based on the Bass model and the value in Eq. (9), the cumulative distribution function is expressed as

$$
F(t)=\frac{1-e^{-(R(t)-R(0))(p+q)}}{1+\frac{q}{p} e^{-(p+q)(R(t)-R(0))}}
$$

Table 1. Parameter explanation

\begin{tabular}{|c|c|}
\hline Parameter & Explanation \\
\hline$f(t)$ & Density function \\
\hline$F(t)$ & Cumulative distribution function \\
\hline$x(t)$ & Current value of alert message \\
\hline$t$ & $\begin{array}{c}\text { Time to disseminate the alert } \\
\text { message }\end{array}$ \\
\hline$(\beta)$ & diffusion parameter \\
\hline$(V(t))$ & Value at current instant \\
\hline$\left(V^{\prime}(t)\right)$ & Value in the next instant \\
\hline$R(t)$ & $\begin{array}{c}\text { Message that is corresponding to } \\
\text { the region }\end{array}$ \\
\hline$N$ & Number of vehicles \\
\hline $\mathrm{N}(t)$ & Vehicle at time $t$ \\
\hline$p_{r}$ & Probability of event \\
\hline
\end{tabular}

Where, $R(t)=t\left(1-\beta \frac{1}{\ln (1-\tau)}\right)$

$$
P_{k}(t)=\operatorname{Pr}(N(t)=k)
$$

In the proposed system, the data diffusion model comprises the closed system with the total count of $N$ vehicles. Initially, one vehicle receives the alert message and the remaining $N-1$ vehicles are unaware of message. The participation of unaware vehicles with the received vehicle through the event discovery model. The probability of staying in state and the shifting state are to be estimated. Based on the estimation values, the alert message is broadcasted among the zones in the previous section.

\subsection{Diffusion model with priority}

The priority assignment to the vehicles based on the generation of urgency message isolates the infected vehicles from the network area. There are three priority values such as susceptible, infected and removed are used in this paper. The initial population value at $t=0$ is $P_{1}(0)=1$ and it is zero otherwise. According to the IEEE 802.11 standard, the emergency data is delivered to $\mathrm{CCH}$ even though the Service Channels $(\mathrm{SCH})$ are available. Based on the urgency condition, the proposed protocol classified the vehicles into the four categories listed in Table 2.

Though the road accidents are occurred infrequently, the $\mathrm{CCH}$ utilization is inefficient which causes the channel wastage due to the fixed durations. Due to these fixed durations, the handling of safety and non-safety message transmissions is inefficient. Hence, in this paper the durations of $\mathrm{CCH}$ and $\mathrm{SCH}$ is dynamically adjusted to improve the channel utilization.

Initially, all the vehicles sense the $\mathrm{CCH}$ first through the broadcast of HELLO message (ID, velocity and location of vehicle) within the communication range. If the channel is in active state, then the vehicle waits until the idle state to avoid the collision. The duration of the vehicle is in

Table 2. Data categories with priority values

\begin{tabular}{|c|c|c|c|}
\hline Data type & $\begin{array}{c}\text { Data } \\
\text { category }\end{array}$ & Priority & Channels \\
\hline $\begin{array}{c}\text { Safety / Control } \\
\text { message }\end{array}$ & Urgent & 1 & CCH \\
\hline $\begin{array}{c}\text { Video and } \\
\text { graphics }\end{array}$ & I & 2 & $\begin{array}{c}\text { SCH1, } \\
\text { SCH2 }\end{array}$ \\
\hline Audio/images & II & 3 & $\begin{array}{c}\text { SCH3, } \\
\text { SCH4 }\end{array}$ \\
\hline Text messages & III & 4 & $\begin{array}{c}\text { SCH5, } \\
\text { SCH6 }\end{array}$ \\
\hline
\end{tabular}


wait state until the channel is transformed into idle state is referred as back-off time. Then, the next phase is to collect the event message regarding to warning during the accidents. The attributes in the warning message includes discover's ID, location, direction, speed, event ID, location and timestamp. When the vehicle receives the repeated warning message, then the single hop neighbors ignore that message after the verification of event ID and the location. During the back-off time, if the vehicle receives the same warning messages, the vehicle drops down the warning message and blocks the back-off process. Sequentially, the multi-hop phase is initiated with the priority validation scheme. The simultaneous decision making through the single and multi-hop transmission with the priority assignment on the basis of urgency accurately provides the remedial actions instantly.

\section{Performance analysis}

In this section, the performance of the proposed UZPDD is investigated against the existing methods of BPAB and UMBP regarding the parameters of Packet Delivery Ratio (PDR), average one-hop delay, throughput, packet loss ratio, routing control overhead with respect to the simulation period, vehicle density and distance with the help of NS-2 simulator [15]. An UMBP developed for disseminate emergency messages and it offered lower emergency message transmission delay and reduce message redundancy. UMBP comprised with novel forwarding node selection scheme which utilizes iterative partition, mini-slot and black-burst to quickly select remote neighbouring node. Single forwarding node is successfully chosen by the asynchronous contention among them. The bidirectional broadcast or multi-directional broadcast and directional broadcast are designed based on position of emergency message sender [8]. An IEEE 802.11based multihop broadcast protocol designed for emergency message dissemination in VANET. These protocols are adopt the binary partition based approach to frequently classified the inside transmission range to obtain the possible segment [2]. The lack of explicit inclusion of design details to support bidirectional broadcast in BPAB, the UMBP and the proposed UZPDD utilizes the multi-directional broadcast strategy to alleviate the issues in existing method. The IEEE $802.11 \mathrm{~b}$ is considered as the base standard and MAC protocol is used for message broadcast. The Manhattan mobility model is adopted which includes horizontal and vertical roads with the random distribution of vehicles on two lane roads. The safety distance between the two vehicles is $10 \mathrm{~m}$ and the overall length of the road segment is $1000 \mathrm{~m}$. Here, the scalability of the network is evaluated by analysing the measures such as throughput, PDR, and packet loss ratio with respect the simulation time, and one hop distance. Moreover, only the authenticated vehicles can able to forward the messages during communication, so the unauthenticated vehicles are blocked for data transmission, which increases the security level

\subsection{Packet delivery ratio}

The ratio of number of packets successfully delivered to the destination to the total number of packets transmitted is referred as Packet Delivery Ratio (PDR) which are linearly increases with the simulation period. In this section, the numerical variations of PDR with the linear increase of simulation period are discussed as shown in Fig. 3.

Fig. 3 shows the variations of PDR for existing $\mathrm{BPAB}, \mathrm{UMBP}$ and proposed UZPDD with respect to the simulation period variations from 10 to 100 ms. In existing methods, the UMBP offers better results compared to $\mathrm{BPAB}$. But, the incorporation of priority validation prior to data transmission improves the PDR values further. For minimum period $(10 \mathrm{~ms})$, the PDR values of UMBP are $55 \%$ and the proposed UZPDD offers $62 \%$. Similarly, they offers 95 and $98 \%$ for maximum period like $100 \mathrm{~ms}$. The comparative analysis shows that the proposed UZPDD improves the PDR values by 11.29 and $3.06 \%$ compared to UMBP for minimum and maximum simulation period respectively.

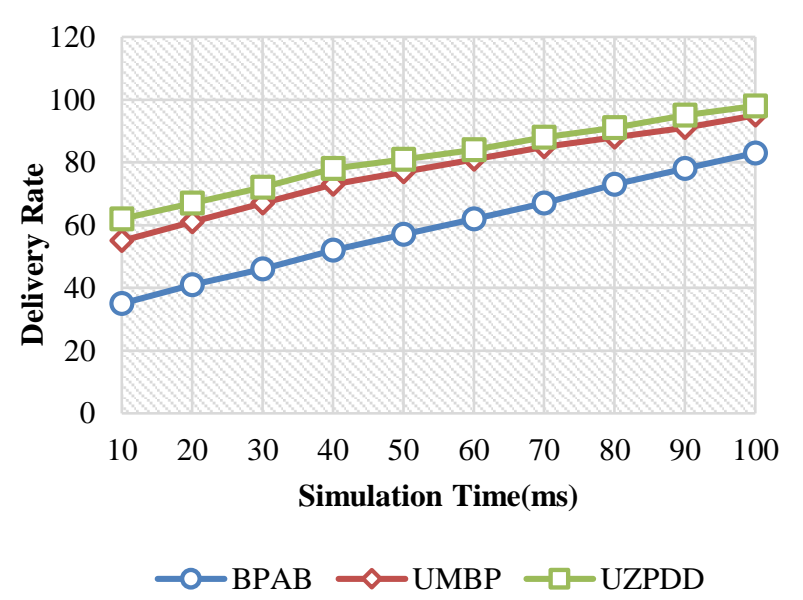

Figure. 3 Packet delivery ratio analysis 


\subsection{Throughput}

The ratio of total number of packets successfully sent to the destination to the time period allotted is referred as throughput which is linearly increases with the simulation period. In this section, the numerical variations of throughput with the linear increase of simulation period are discussed as shown in Fig. 4. Fig. 4 shows the variations of throughput for existing $\mathrm{BPAB}, \mathrm{UMBP}$ and proposed UZPDD with respect to the simulation period variations from 10 to $100 \mathrm{~s}$. In existing methods, the UMBP offers better results compared to BPAB. But, the incorporation of priority validation prior to data transmission improves the PDR values further. For minimum period (10s), the throughput values of UMBP are $653 \mathrm{kbps}$ and the proposed UZPDD offers 684 kbps. Similarly, they offer 3477 and 3543 kbps for maximum period like 100s. The comparative analysis shows that the proposed UZPDD improves the throughput values by 4.53 and $1.86 \%$ compared to UMBP for minimum and maximum simulation period respectively

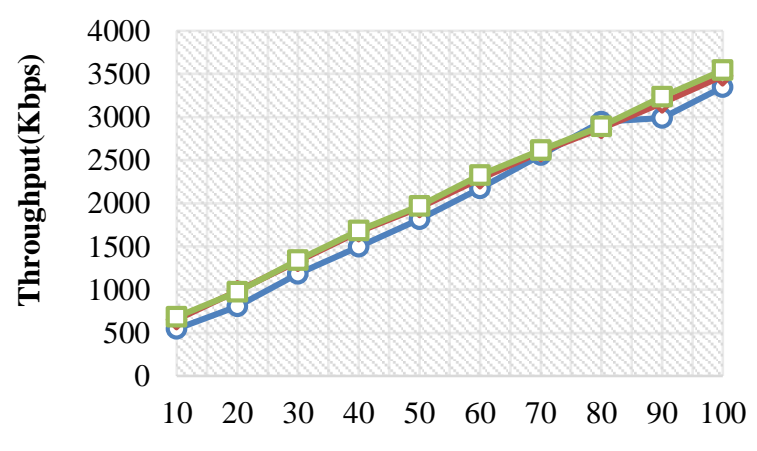

Simulation Time (ms)

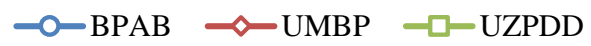

Figure. 4 Throughput analysis

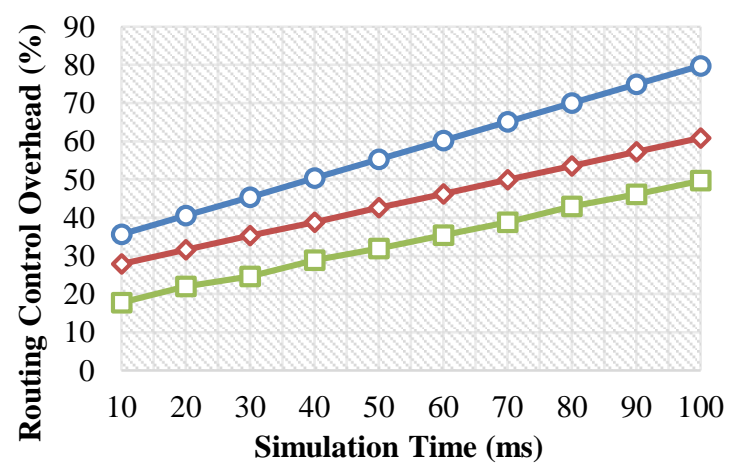

$\multimap$ ВPAB $\multimap$ UMBP $-\square$ UZPDD

Figure. 5 Routing control overhead analysis

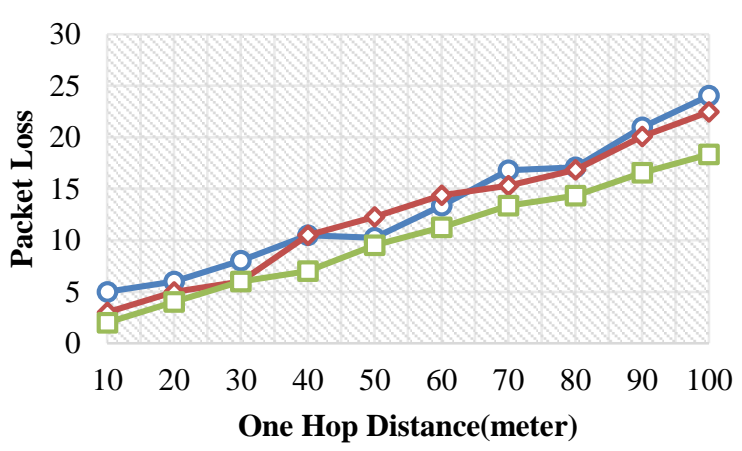

- - ВPAB $\prec$ UMBP $\square-$ UZPDD

Figure. 6 Packet loss ratio analysis

\subsection{Routing control overhead}

In this section, the numerical variations of overhead with the linear increase of simulation period are discussed as shown in Fig. 5.

Fig. 5 shows the variations of overhead for existing $\mathrm{BPAB}, \mathrm{UMBP}$ and proposed UZPDD with respect to the simulation period variations from 10 to $100 \mathrm{~ms}$. In existing methods, the UMBP offers minimum overhead compared to BPAB. But, the incorporation of priority validation prior to data transmission reduces the overhead values further. For minimum period $(10 \mathrm{~ms})$, the overhead values of UMBP and UZPDD are 27.869 and $17.769 \%$. Similarly, they offers 60.878 and $49.66 \%$ for maximum period like $100 \mathrm{~ms}$. The comparative analysis shows that the proposed UZPDD reduces the overhead by 36.24 and $18.43 \%$ compared to UMBP for minimum and maximum simulation period respectively.

\subsection{Packet loss ratio}

The ratio of number of packets lossed to the destination to the total number of packets transmitted is referred as Packet Loss Ratio which is linearly increases with the increase in distance. In this section, the numerical variations of loss ratio with the linear increase of distance are discussed as shown in Fig. 6.

Fig. 6 shows the variations of packet loss ratio for existing BPAB, UMBP and proposed UZPDD with respect to the distance variations from 10 to $100 \mathrm{~m}$. In existing methods, the UMBP offers minimum compared to BPAB. But, the incorporation of priority validation prior to data transmission reduces the loss ratio further. For minimum distance $(10 \mathrm{~m})$, the loss ratio for UMBP is $3 \%$ and the proposed UZPDD offers $2 \%$. 


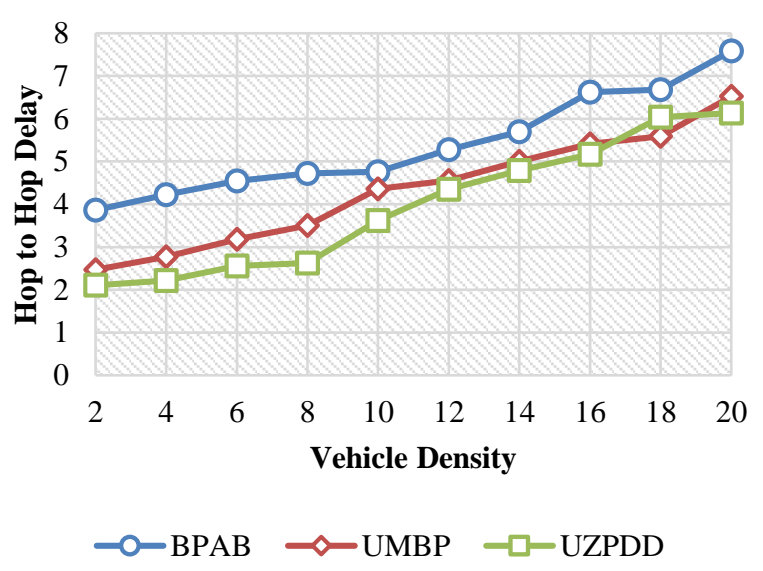

Figure. 7 Average one-hop delay analysis

Similarly, they offer 22.46 and $18.34 \%$ for maximum distance like $100 \mathrm{~m}$. The comparative analysis shows that the proposed UZPDD reduces the loss ratio values by 33.33 and $18.34 \%$ compared to UMBP for minimum and maximum distance respectively.

\subsection{Average one hop delay}

The time duration for the transmission of emergency message to the single-hop neighbours from the source is referred as one hop delay which is linearly decreases with the vehicle density. In this section, the numerical variations of average one hop delay with the linear increase of distance are discussed as shown in Fig. 7.

Fig. 7 shows the variations of average one hop delay for existing BPAB, UMBP and proposed UZPDD with respect to the vehicle density of 2 to 20. In existing methods, the UMBP offers better results compared to BPAB. But, the incorporation of priority validation prior to data transmission reduces the delay further. For minimum density (2), the delay values of UMBP are $2.4678 \mathrm{~ms}$ and the proposed UZPDD offers $2.104 \mathrm{~ms}$. similarly, they offer 6.5203 and $6.1332 \mathrm{~ms}$ for maximum density 20 .

\section{Conclusion}

In this paper, the issues affected the broadcasting of messages among the vehicle nodes to handle the emergency scenarios such as limited bandwidth, minimum contention window size and the mobility of vehicular nodes are reviewed. This paper proposed the suitable data diffusion model with the emergency message transfer and the clustering of neighborhood nodes based on the zones. On the basis of the location of the emergency-message senders, the creation of zone such as Red Orange and Yellow (ROY) is created to partition the vehicle broadcasting directions. The comparative analysis shows that the proposed UZPDD reduces the delay values by 14.74 and $5.93 \%$ compared to UMBP for minimum and maximum vehicle density respectively. The adjustments in urgency factor by the vehicle on the basis of the emergency handled the traffic target areas seamlessly. The analysis and simulation results showed that the proposed diffusion model improvement in delay, message overhead and reception rate compared to the existing models.

In future, the accuracy will be achieved with far less delay and effectiveness will be added on overall performance.

\section{References}

[1] T. H. Luan, X. Ling, and X. Shen, "MAC in motion: Impact of Mobility on the MAC of Drive-Thru Internet", IEEE Transactions on Mobile Computing, Vol. 11, No, 2, pp. 305-319, 2012.

[2] J. Sahoo, E. H.-K. Wu, P. K. Sahu, and M. Gerla, "Binary-partition-assisted MAC-layer Broadcast for Emergency Message Dissemination in VANETs", IEEE Transactions on Intelligent Transportation Systems, Vol. 12, No. 3, pp. 757-770, 2011.

[3] R. Zhang, X. Cheng, Q. Yao, C.-X. Wang, Y. Yang, and B. Jiao, "Interference Graph-based Resource-sharing Schemes for Vehicular Networks", IEEE Transactions on Vehicular Technology, Vol. 62, No. 8, pp. 4028-4039, 2013.

[4] X. Cheng, Q. Yao, C. X. Wang, B. Ai, G. L. Stuber, D. Yuan, and B. L. Jiao, "An Improved Parameter Computation Method for a MIMO V2V Rayleigh Fading Channel Simulator Under Non-Isotropic Scattering Environments", IEEE Communications Letters, Vol. 17, No. 2, pp. 265-268, 2013.

[5] X. Cheng, C. X. Wang, B. Ai, and H. Aggoune, "Envelope Level Crossing Rate and Average Fade Duration Of Nonisotropic Vehicle-ToVehicle Ricean Fading Channels", IEEE Transactions on Intelligent Transportation Systems, Vol. 15, No. 1, pp. 62-72, 2014.

[6] S. M. Tornell, C. T. Calafate, J. C. Cano, P. Manzoni, M. Fogue, and F. J. Martinez, "Implementing and Testing a Driving Safety Application for Smartphones Based on the eMDR Protocol", In: Proce. of the International Conference on Wireless Days (WD), IFIP, Dublin, Ireland, pp. 1-3, 2012. 
[7] M. Fogue, P. Garrido, F. J. Martinez, J. C. Cano, C. T. Calafate, and P. Manzoni, "An Adaptive System Based on Roadmap Profiling to Enhance Warning Message Dissemination in VANETs", IEEE/ACM Transactions on Networking, Vol. 21, No. 3, pp. 883-895, 2013.

[8] Y. Bi, H. Shan, X. S. Shen, N. Wang, and H. Zhao, "A Multi-Hop Broadcast Protocol For Emergency Message Dissemination in Urban Vehicular Ad Hoc Networks", IEEE Transactions on Intelligent Transportation Systems, Vol. 17, No. 3, pp. 736-750, 2016.

[9] X. Cheng, L. Yang, and X. Shen, "D2D for Intelligent Transportation Systems: A Feasibility Study", IEEE Transactions on Intelligent Transportation Systems, Vol. 16, No. 4, pp. 1784-1793, 2015.

[10] C. Suthaputchakun, M. Dianati, and Z. Sun, "Trinary Partitioned Black-Burst-Based Broadcast Protocol for Time-Critical Emergency Message Dissemination in VANETs", IEEE transactions on vehicular technology, Vol. 63, No. 6, pp. 2926-2940, 2014.

[11] X. Ma, J. Zhang, X. Yin, and K. S. Trivedi, "Design and Analysis of a Robust Broadcast Scheme for VANET Safety-Related Services", IEEE Transactions on Vehicular Technology, Vol. 61, No. 1, pp. 46-61, 2012.

[12] H. A. Omar, W. Zhuang, and L. Li, "VeMAC: A TDMA-based MAC Protocol for Reliable Broadcast in VANETs", IEEE Transactions on Mobile Computing, Vol. 12, No. 9, pp. 17241736, 2013.

[13] H. A. Omar, W. Zhuang, A. Abdrabou, and L. $\mathrm{Li}$, "Performance Evaluation of VeMAC Supporting Safety Applications in Vehicular Networks", IEEE Transactions on Emerging Topics in Computing, Vol. 1, No. 1, pp. 69-83, 2013.

[14] H. T. Cheng, H. Shan, and W. Zhuang, "Infotainment and Road Safety Service Support in Vehicular Networking: From a Communication Perspective", Mechanical Systems and Signal Processing, Vol. 25, No. 5, pp. 2020-2038, 2011.

[15] T. N. Simulator-ns-2. Available: http://www.isi.edu/nsnam/ns/. 